\title{
BMJ Open Risk of dyslipidaemia with antipsychotic drug treatment in Chinese inpatients with mental illness: a hospital-based cohort study
}

Qiuyue Ma, ${ }^{1}$ Fude Yang, ${ }^{2}$ Botao $\mathrm{Ma},{ }^{2}$ Wenzhan Jing, ${ }^{1}$ Jue Liu, ${ }^{1}$ Moning Guo, ${ }^{3}$ Juan Li, ${ }^{4}$ Zhiren Wang, ${ }^{2}$ Min Liu (iD) ${ }^{1}$

To cite: Ma Q, Yang F, Ma B, et al. Risk of dyslipidaemia with antipsychotic drug treatment in Chinese inpatients with mental illness: a hospitalbased cohort study. BMJ Open 2021;11:e043259. doi:10.1136/ bmjopen-2020-043259

- Prepublication history for this paper is available online. To view these files, please visit the journal online (http://dx.doi. org/10.1136/bmjopen-2020043259).

Received 29 July 2020 Revised 22 December 2020 Accepted 13 January 2021

\section{Check for updates}

(c) Author(s) (or their employer(s)) 2021. Re-use permitted under CC BY-NC. No commercial re-use. See rights and permissions. Published by BMJ.

${ }^{1}$ Department of Epidemiology and Biostatistics, Peking University School of Public Health, Beijing, China

${ }^{2}$ Peking University Huilonguan Clinical Medical School, Beijing Huilongguan Hospital, Beijing, China

${ }^{3}$ Beijing Municipal Commission of Health and Family Planning Policy Research Center, Beijing Municipal Commission of Health and Family Planning Information Center, Beijing, China

${ }^{4}$ Beijing Geriatric Hospital, Beijing, China

\section{Correspondence to}

Dr Min Liu;

liumin@bjmu.edu.cn and

Dr Fude Yang;

yangfd200@126.com

\section{ABSTRACT}

Objectives To determine the association of long-term use of antipsychotics with the risk of dyslipidaemia.

Design A hospital-based cohort study.

Setting Electronic health record data of adult mental health inpatients in all 19 specialised psychiatric hospitals in Beijing from 1 January 2005 to 31 December 2018 was obtained.

Participants Participants were inpatients aged 18 years or older with at least two admissions, excluding those with diagnosed dyslipidaemia and fatty liver at the first admission. We included 22329 adult inpatients with no dyslipidaemia and fatty liver at baseline. The exposure was antipsychotics use, defined as antipsychotics prescription in the treatment procedures of medical record preceding dyslipidaemia diagnosis during the follow-up period. 15930 (71.34\%) had antipsychotics use, and 6399 (28.66\%) never had antipsychotics use. We used the length of follow-up as proxy for the duration of antipsychotics exposure.

Primary outcome measures The primary outcome was newly recorded dyslipidaemia defined by International Classification of Diseases, 10th Revision codes.

Results 4069 inpatients had newly recorded dyslipidaemia during 73418.07 person-years, the incidence rate was 5.54 per 100 person-years. The incidence rate was 7.22 per 100 person-years in the exposed group and 3.43 per 100 person-years in the unexposed group. Results of multivariate analysis showed that antipsychotics use was associated with higher risk of dyslipidaemia (adjusted HR, aHR 2.41, $95 \% \mathrm{Cl} 2.24$ to $2.59, \mathrm{p}<0.001$ ), regardless of the duration of antipsychotics use. Inpatients aged 18-29 years had higher risk of dyslipidaemia (aHR $3.38,95 \% \mathrm{Cl} 2.77$ to 4.12 , $p=0.004$ ) than those in other age groups. Inpatients without hypertension had substantially higher risk of dyslipidaemia after antipsychotic exposure.

Conclusions Both short-term and long-term antipsychotics use was associated with higher risk of dyslipidaemia among Chinese inpatients with mental illness. Dyslipidaemia was especially prominent in young patients and those without hypertension.

\section{INTRODUCTION}

Mental illness is a major contributor to the global burden of disease. ${ }^{1}$ Compared with
Strengths and limitations of this study

- This study was a large hospital-based cohort study, used electronic health record data of all individuals with inpatient psychiatric hospitalisation among 19 hospitals in Beijing.

- The long-term follow-up enabled us to detect the long-term effect of antipsychotic treatment.

- One of the limitations is that some dyslipidaemia risk factors such as body weight of the inpatients are not available from the electronic health record data.

the general population, mental health patients, such as schizophrenia, bipolar disorder and depression, have twofold to threefold increased mortality risk, corresponding to the $10-25$ years shortened life expectancy. ${ }^{2-4}$ As in the general population, cardiovascular diseases (CVDs) are the most common cause of death in patients with severe mental illness (SMI). ${ }^{1}$ Dyslipidaemia is one of the major risk factors of CVDs among Chinese adults. ${ }^{5}$ The overall dyslipidaemia prevalence was $43 \%$ among middle-aged and older Chinese adults. ${ }^{6}$ Mental health patients are more likely to develop high rate of dyslipidaemia, which is attributed to an interaction between the disease itself, lifestyle factors, physical health inequalities and treatment effects. $^{7-9}$ It was found that about half the patients with first-episode schizophrenia spectrum disorders had dyslipidaemia, and the prevalence in patients with schizophrenia was $66 \% .^{810}$

Antipsychotic medications contribute to the development of metabolic syndrome, including dyslipidaemia. ${ }^{11}$ The introduction of chlorpromazine into clinical practice in 1950 s sparked a revolution in the treatment of schizophrenia. ${ }^{12}$ 
Antipsychotics form the mainstay of treatment for schizophrenia patients, which maintain symptom relief, reduce the risk of relapse and improve clinical outcome. ${ }^{11} 13$ Given that the majority of schizophrenia patients face chronic, even lifelong treatment with antipsychotics, the risk of dyslipidaemia is major concern for patients with long-term antipsychotics treatment. ${ }^{14}$ Previous study showed that dyslipidaemia was more prevalent in people receiving antipsychotic treatment. ${ }^{15}$

Nowadays, antipsychotics are used to treat a broad range of symptoms and disorders. ${ }^{12} 1617$ There is need for longitudinal studies to establish the long-term contribution of antipsychotics to dyslipidaemia in mental health inpatients. Recent meta-analysis of 100 randomised controlled trials (RCTs) shows that total cholesterol (TC), LDL cholesterol and triglyceride (TG) concentrations increased with olanzapine and clozapine. ${ }^{11}$ However, a major issue in RCTs is the selection of patients, as those with physical comorbidity are often excluded. ${ }^{18}$ According to a retrospective cohort study conducted in Chinese first-episode schizophrenia inpatients, lipid abnormalities markers were significantly elevated after an average of 22.7 days of antipsychotic exposure. ${ }^{19}$ However, a longterm follow-up is preferred to detect the impact of antipsychotics on dyslipidaemia.

In this study, we conducted a hospital-based cohort study, to determine the association of long-term use of antipsychotics with the risk of dyslipidaemia in Chinese mental health inpatients. The hypothesis is patients with long-term use of antipsychotics had higher risk of dyslipidaemia compared with patients never used antipsychotics.

\section{MATERIALS AND METHODS \\ Study design and participants}

This is a multicentre hospital-based cohort study. Data were obtained from Beijing Municipal Commission of Health and Family Planning Information Centre, which included electronic health record (EHR) data of adult mental health inpatients in all 19 specialised psychiatric hospitals in Beijing, China, from 2005 to 2018. We collected demographic characteristics, discharge diagnoses, treatment procedures of inpatients from the EHR dataset. Discharge diagnoses were made by physicians according to the 10th revision of the International Classification of Diseases, 10th Revision (ICD-10) ${ }^{20}$ Treatment information was recorded by physicians in the EHR during the clinical practice.

Eligible participants were inpatients aged 18 years or older with mental illness who discharged from the 19 specialised psychiatric hospitals in Beijing between 1 January 2005 and 31 December 2018. Inpatients who had only one admission record were excluded because at least two admissions were needed for follow-up. Furthermore, inpatients with diagnosed dyslipidaemia (ICD-10 code: E78) or fatty liver (ICD-10 code: K76.0) at the first admission were excluded.

We identified 115157 mental health inpatients who were aged 18 years and older between 1 January 2005 and 31 December 2018. After excluding 87759 inpatients with only one admission record during the study period, 3716

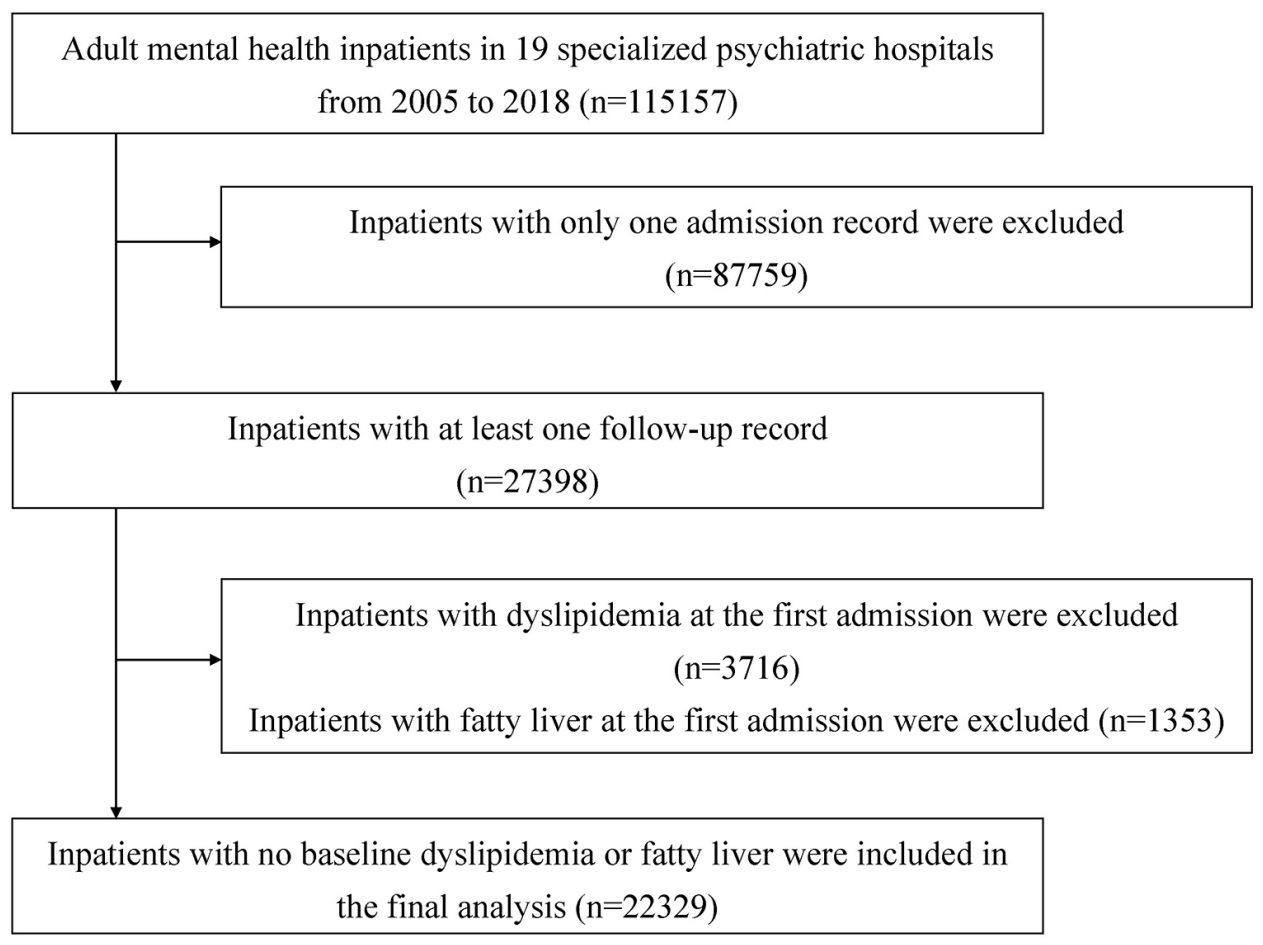

Figure 1 Flow diagram of the study participants. 
with dyslipidaemia at the first admission, and 1353 with fatty liver at the first admission, we included 22329 adult inpatients in the cohort for final analysis (figure 1). The diagnoses of inpatients were schizophrenia, schizotypal and delusional disorders (F20-F29), mood disorders (F30-F39) and other mental illness.

\section{Exposure}

The exposure in this study was antipsychotics use, defined as antipsychotics prescription in the treatment procedures of medical record preceding dyslipidaemia diagnosis. Unexposed was defined as no antipsychotics prescription in the medical record during the entire study period. The index date for the exposed group was the admission date of their first medical record with an antipsychotics prescription preceding dyslipidaemia diagnosis. The index date for the unexposed group was the admission date of their first medical record.

\section{Outcome}

In this study, the main outcome was newly recorded dyslipidaemia, defined as ICD-10 codes E78.0-E78.9 (including pure hypocholesterolaemia, pure hyperglyceridaemia, mixed hyperlipidaemia, hyperchylomicronaemia, other hyperlipidaemia, unspecified hyperlipidaemia, lipoprotein deficiency, other disorder of lipoprotein metabolism, unspecified disorder of lipoprotein metabolism) in the discharge diagnosis. All inpatients underwent routine blood tests when they were admitted in hospitals. TC, low-density lipoprotein-cholesterol (LDL-C), high-density lipoprotein-cholesterol (HDL-C) and TG (TG) were examined. The cut-off values for higher TC: $\geq 6.22 \mathrm{mmol} / \mathrm{L}$, higher LDL-C: $\geq 4.14 \mathrm{mmol} / \mathrm{L}$, lower HDL-C: $<1.04 \mathrm{mmol} / \mathrm{L}$, higher TG: $\geq 2.26 \mathrm{mmol} / \mathrm{L} .{ }^{21}$ Physicians diagnosed dyslipidaemia according to blood examination, and recorded in the medical record using ICD-10 code. Up to 40 diagnostic codes can be recorded for each inpatient in the medical records, thus all detected dyslipidaemia cases were recorded. Follow-up began on index date and ceased when inpatients had a newly recorded dyslipidaemia or on the discharge date of the last admission. In this study, we used the length of follow-up as proxy for the duration of antipsychotics exposure.

\section{Covariates}

We identified several baseline covariates from the medical records of index date to include in the multivariable models: gender (men, women), age (divided into six groups: $18-29,30-39,40-49,50-59,60-69$ and $\geq 70$ ), ethnic origin (Han, others), marital status (never married, married, others), payment (new rural cooperative medical scheme, urban employees basic medical insurance (UEBMI), urban resident basic medical insurance (URBMI), other insurance, out-of-pocket (OOP) expenditures), hospital level (third level, second level). Comorbid conditions were also identified according to ICD-10 codes: hypertension (I10-I15, including essential hypertension, hypertensive heart disease, hypertensive renal disease, hypertensive heart and renal disease, secondary hypertension), diabetes mellitus (E10-E14, including insulin-dependent diabetes mellitus, noninsulin-dependent diabetes mellitus, malnutrition-related diabetes mellitus, other specified diabetes mellitus, unspecified diabetes mellitus). The number of admissions during the follow-up period was divided into two groups ( 2 and $\geq 3$ ).

\section{Statistical analysis}

Age of participants was described by median and IQRs. Baseline characteristics were described as proportions, and the $\chi^{2}$ test was used to compare the distribution of baseline characteristics between exposed and unexposed group. We calculated the follow-up person-years, and incidence rate for dyslipidaemia, using number of newly recorded dyslipidaemia divided by follow-up person-years for exposed and unexposed groups, respectively. We also calculated the incidence rate for dyslipidaemia by follow-up time. We used univariable and multivariable Cox regression models to estimate crude HR (cHR), adjusted HR (aHR) and their 95\% CI. The multivariable analysis was adjusted for covariates at cohort entry (gender, age groups, ethnic origin, marital status, payment, hospital level, hypertension, diabetes mellitus) and number of admissions during the follow-up period.

In the subgroup analysis, we divided inpatients into different subgroups according to baseline characteristics. Cox regression models were used to estimate cHRs, aHRs and their 95\% CIs. We examined the association between antipsychotics and the risk of dyslipidaemia after adjusting for other covariates. We did a sensitivity analysis excluding inpatients with mental and behavioural disorder due to the use of alcohol (ICD-10 code: F10). All analyses were performed with SPSS Statistics for Windows, V.19.0 (IBM), and Stata MP V.14. All p values are two sided with significance set as $\mathrm{p}<0.05$.

\section{Consent to participate}

All identifiable information was removed.

\section{Patient and public involvement}

Patients and the public were not involved in the design and conduct of the study. But the study findings will be disseminated through multiple channels including publication, meetings, conferences and social media.

\section{RESULTS}

We included 22329 adult mental health inpatients in this cohort. The median age of all participants was 40 years (IQR 27-54). 52.12\% of the participants were female, $49.39 \%$ were between 18 and 39 years old, $93.27 \%$ were Han ethnic, $47.95 \%$ were married, $42.49 \%$ had insurance of UEBMI, $79.96 \%$ were from third level hospitals. $12.93 \%$ of participants had hypertension, $8.37 \%$ had diabetes, $50.20 \%$ had three or more admissions. 10435 (46.73\%) inpatients had schizophrenia, schizotypal and 
Table 1 Baseline characteristics of antipsychotics exposed and unexposed inpatients

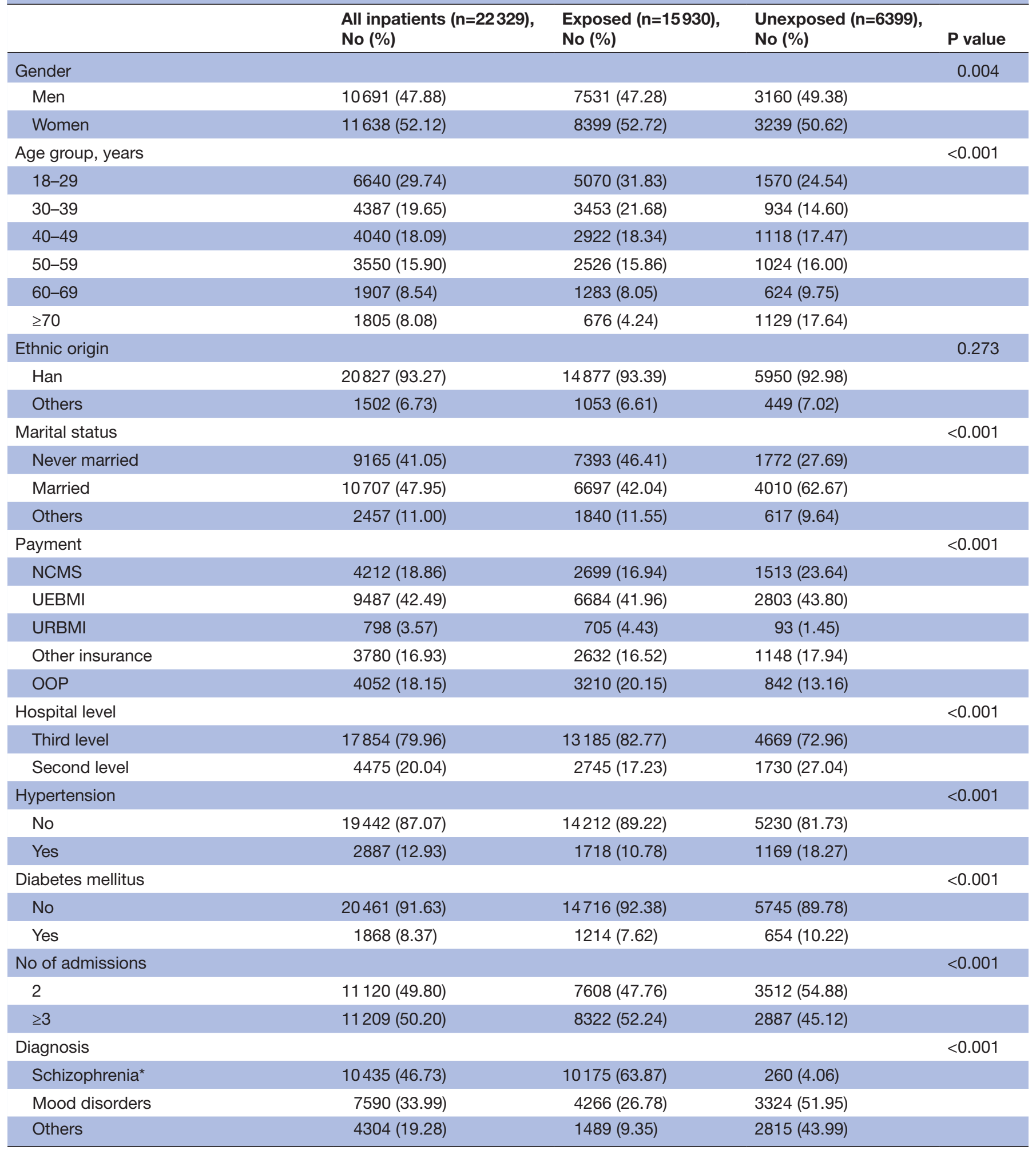

*Schizophrenia, schizotypal and delusional disorders.

NCMS, new rural cooperative medical scheme; OOP, out-of-pocket; UEBMI, urban employees basic medical insurance; URBMI, urban resident basic medical insurance.

delusional disorders, $7590(33.99 \%)$ had mood disorders, 4304 (19.28\%) had other mental illness (table 1).

Among all the inpatients, 15930 (71.34\%) had antipsychotics use, and 6399 (28.66\%) never had antipsychotics use. Compared with the unexposed inpatients, inpatients with antipsychotics use were more likely to be female $(\mathrm{p}=0.004)$, young $(\mathrm{p}<0.001)$, unmarried $(\mathrm{p}<0.001)$, from third level hospitals $(\mathrm{p}<0.001)$, have three or more 
Table 2 Incidence rate and risk of dyslipidaemia by follow years

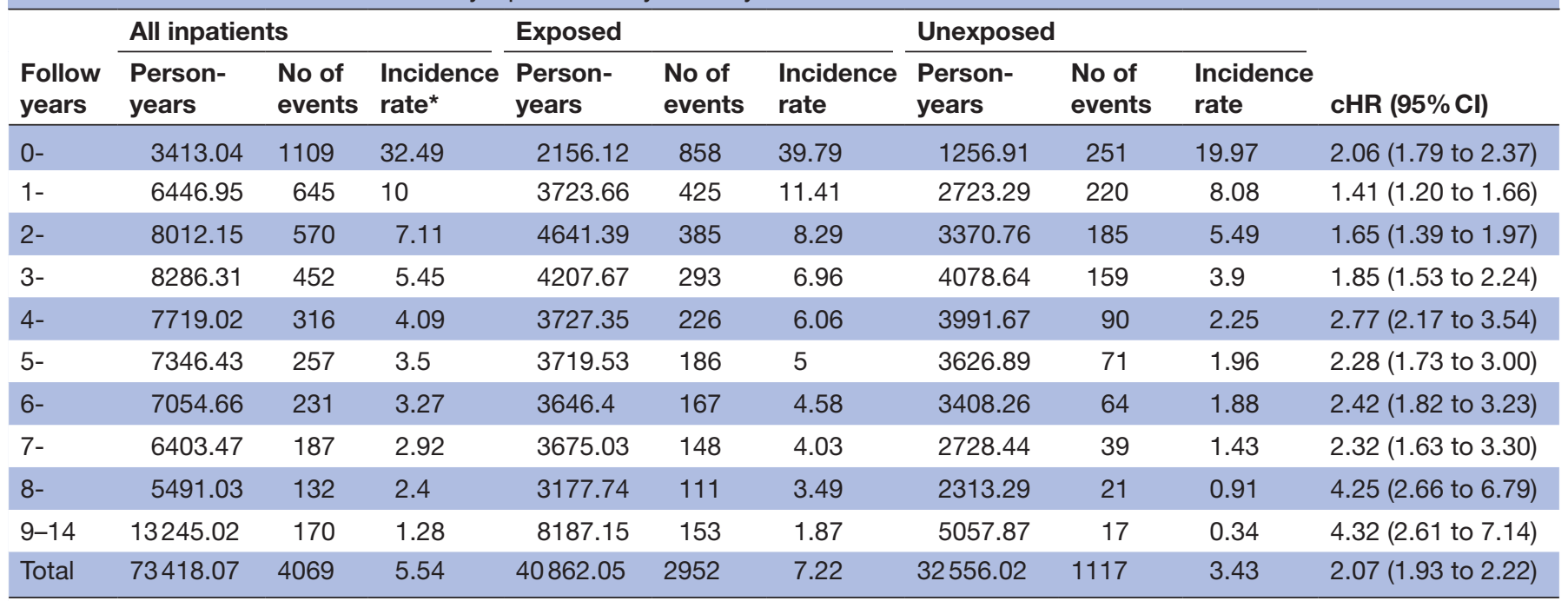

${ }^{*}$ Per 100 person-years.

cHR, crude HR.

admissions $(\mathrm{p}<0.001)$, have schizophrenia, schizotypal and delusional disorders $(p<0.001)$ and less likely to have hypertension $(p<0.001)$, diabetes $(p<0.001)$, mood disorders and other mental illness $(\mathrm{p}<0.001$, table 1$)$.

A total of 4069 inpatients had newly recorded dyslipidaemia during 73418.07 person-years follow-up, the incidence rate was 5.54 per 100 person-years. In the exposed group, 2952 had newly recorded dyslipidaemia during 40862.05 person-years, the incidence rate was 7.22 per 100 person-years. In the unexposed group, 1117 had newly recorded dyslipidaemia during 32556.02 personyears, the incidence rate was 3.43 per 100 person-years. The highest incidence rate was observed in the first year of follow-up for exposed and unexposed group. The incidence rate declined as follow-up time increased. In the unadjusted Cox model, exposure to antipsychotics was associated with increased risk of dyslipidaemia (cHR 2.07, $95 \%$ CI 1.93 to 2.22, $\mathrm{p}<0.001$, table 2).

Results of multivariate analysis showed that antipsychotics use was associated with higher risk of dyslipidaemia (aHR 2.41, 95\% CI 2.24 to 2.59, p<0.001), regardless of the duration of antipsychotics use. The aHR was 2.25 (95\% CI 1.93 to 2.61, $\mathrm{p}<0.001)$ in the first year of follow-up. Inpatients who had received long-term antipsychotics use had much higher risk of dyslipidaemia. The aHR was 4.58 (95\% CI 2.82 to $7.45, \mathrm{p}<0.001$ ) when the follow-up time reached 8years, and 4.38 (95\% CI 2.58 to 7.41, $\mathrm{p}<0.001)$ when the follow-up time reached 9 years (figure 2).

In the subgroup analyses, the risk of dyslipidaemia was significantly higher in the antipsychotics exposed group regardless of the gender, age, ethnic origin, marital status of participants. Inpatients aged 18-29 years had higher aHR $(3.38,95 \%$ CI 2.77 to $4.12, \mathrm{p}=0.004)$ than those in other age groups. Inpatients from third level hospitals had higher aHR $(2.77,95 \%$ CI 2.54 to $3.03, \mathrm{p}<0.001)$ than those from second level hospitals. Among those who did not have hypertension at the baseline, a much higher aHR (2.47, 95\% CI 2.27 to 2.68, $\mathrm{p}=0.030$ ) was observed than those who had hypertension at the baseline (aHR $2.04,95 \%$ CI 1.74 to 2.40 ). A similar trend was observed between inpatients with diabetes and those without, although the difference was not significant. Those who did not have diabetes at the baseline had higher aHR (2.45, $95 \%$ CI 2.26 to $2.65, \mathrm{p}=0.304$ ) than those had diabetes at the baseline (aHR 2.20, 95\% CI 1.80 to 2.67). Compared with inpatients with three or more admissions, those with two admissions had higher aHR for dyslipidaemia (aHR $3.28,95 \%$ CI 2.94 to $3.67, \mathrm{p}<0.001$, figure 3 ).

In the sensitivity analysis, exclusion of those who had mental and behavioural disorder due to use of alcohol did not alter the association between antipsychotics and

\begin{tabular}{|c|c|c|c|c|c|c|}
\hline \multirow{2}{*}{$\begin{array}{l}\text { Follow } \\
\text { years }\end{array}$} & \multicolumn{2}{|c|}{ Exposed } & \multicolumn{2}{|c|}{ Unexposed } & & \multirow{2}{*}{$\begin{array}{r}\text { Adjusted hazard } \\
\text { ratio }(95 \% \text { CI })\end{array}$} \\
\hline & Number & Person-years & Number & Person-years & & \\
\hline $0-$ & 858 & 2156.12 & 251 & 1256.91 & $\bullet$ & $2.25(1.93,2.61)$ \\
\hline $1-$ & 425 & 3723.66 & 220 & 2723.29 & $\bullet$ & $1.41(1.19,1.68)$ \\
\hline $2-$ & 385 & 4641.39 & 185 & 3370.76 & + & $1.78(1.48,2.15)$ \\
\hline 3- & 293 & 4207.67 & 159 & 4078.64 & $\bullet$ & $1.88(1.53,2.32)$ \\
\hline 4- & 226 & 3727.35 & 90 & 3991.67 & $\rightarrow$ & $2.48(1.90,3.22)$ \\
\hline $5-$ & 186 & 3719.53 & 71 & 3626.89 & $\leftarrow$ & $2.06(1.52,2.80)$ \\
\hline $6-$ & 167 & 3646.40 & 64 & 3408.26 & $\rightarrow$ & $2.15(1.57,2.94)$ \\
\hline 7- & 148 & 3675.03 & 39 & 2728.44 & $\rightarrow$ & $2.01(1.36,2.95)$ \\
\hline 8- & 111 & 3177.74 & 21 & 2313.29 & - & $4.58(2.82,7.45)$ \\
\hline $9-14$ & 153 & 8187.15 & 17 & 5057.87 & - & $4.38(2.58,7.41)$ \\
\hline Total & 2952 & 40862.05 & 1117 & 32556.02 & - & $2.41(2.24,2.59)$ \\
\hline
\end{tabular}

Figure 2 Risk of dyslipidaemia for antipsychotics exposed inpatients by follow years (adjusted for gender, age groups, ethnic origin, marital status, payment, hospital level, hypertension, diabetes mellitus and number of admissions). 


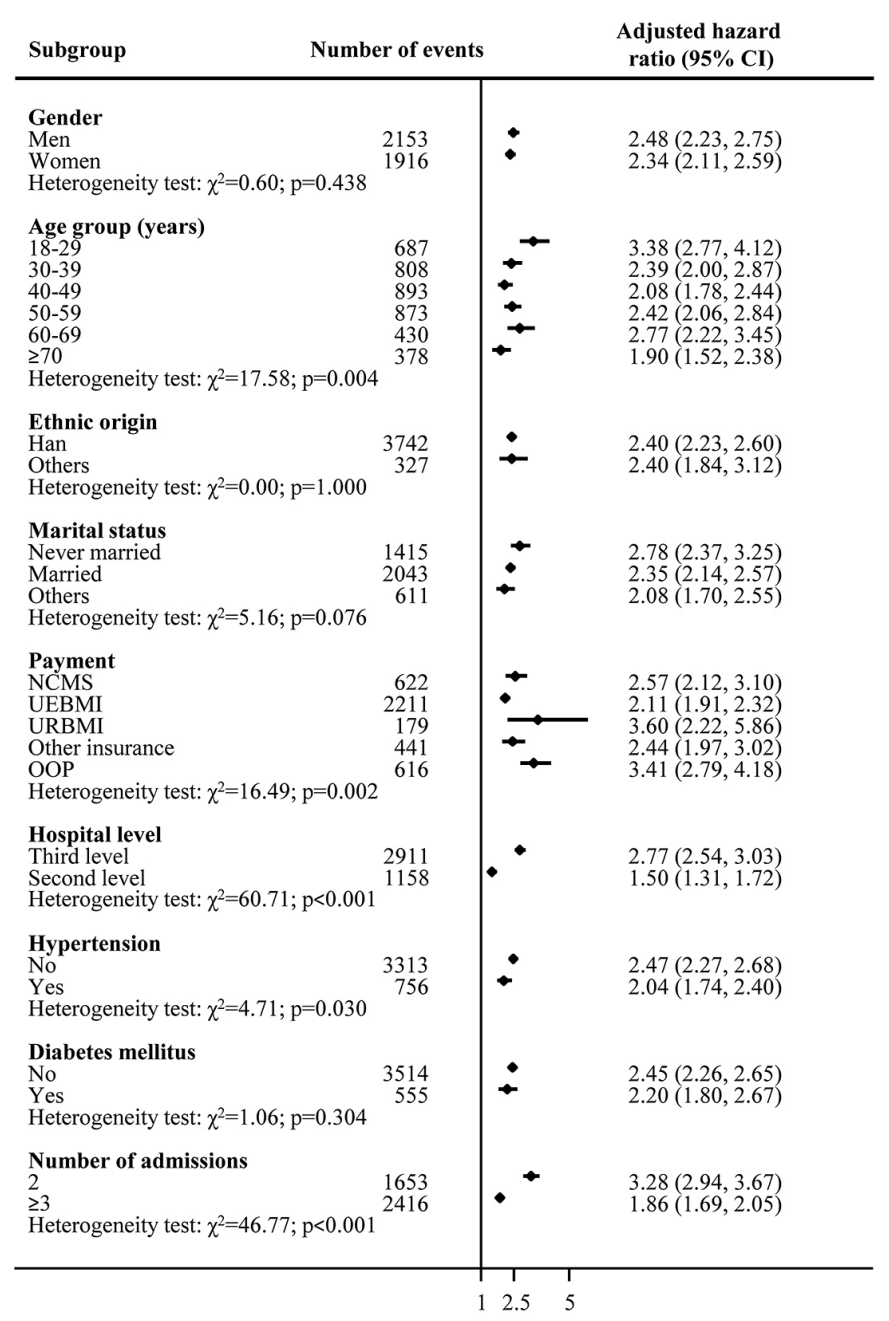

Figure 3 Subgroup analyses of association between antipsychotics and the risk of dyslipidaemia (adjusted for gender, age groups, ethnic origin, marital status, payment, hospital level, hypertension, diabetes mellitus and number of admissions).

risk of dyslipidaemia (aHR 2.48, 95\% CI 2.29 to 2.68, $\mathrm{p}<0.001)$.

\section{DISCUSSION}

To our knowledge, this is the largest hospital-based cohort study conducted among Chinese mental health inpatients, which had long-term follow-up period to detect the longterm impact of antipsychotics on dyslipidaemia. In this study, we found that the incidence rate of dyslipidaemia was 5.54 per 100 person-years among mental health inpatients. Antipsychotics use was associated with a twofold higher incidence rate of dyslipidaemia. The incidence rate of dyslipidaemia was 7.22 and 3.43 per 100 personyears in patients who used antipsychotics and those who never used antipsychotics. Researchers examined the incidence of hyperlipidaemia among patients with major depressive disorder (MDD) and bipolar disorder, the annual incidence of hyperlipidaemia was $3.62 \%$ for MDD and $4.37 \%$ for bipolar disorder. ${ }^{22}{ }^{23}$ The overall dyslipidaemia incidence rate in our study was slightly higher than the results from above-mentioned studies. One of the reasons that could have caused the different incidence rates was the different study design. In our study, we included patients with MDD and bipolar disorder, as well as those with schizophrenia, schizoaffective disorder. Moreover, our study had a long-term follow-up, which benefits the detection of long-term outcomes.

Our findings showed that antipsychotics use was associated with an increased risk of dyslipidaemia. After adjusting for all covariates, results from Cox regression showed that inpatients who used antipsychotics were 2.41 times more likely for dyslipidaemia compared with those never used antipsychotics. This result was consistent with previous studies. ${ }^{1124}$ Vancampfort $e t a l^{24}$ conducted a meta-analysis of metabolic syndrome and its components among people with SMI. They found that patients receiving all individual antipsychotics were at higher metabolic syndrome risk when compared with those who never received antipsychotics. A recent metaanalysis including 100 RCTs observed increases in TC, LDL cholesterol and TG concentrations with quetiapine, olanzapine and clozapine, suggesting that the most efficacious antipsychotics were associated with the greatest metabolic disturbance. ${ }^{11}$ In this study, antipsychotics as a group was associated with higher risk of dyslipidaemia among mental health inpatients. However, it is worth noting that inpatients from exposed group were more likely to have schizophrenia, schizotypal and delusional disorders, which might be related to the higher risk for dyslipidaemia. Furthermore, previous study showed that antipsychotics differed substantially in side effects. ${ }^{25}$ Dyslipidaemia risk related to specific antipsychotics remains a topic that warrant further research.

In our study, both short-term and long-term antipsychotics use were associated with higher risk of dyslipidaemia. Zhai et $a l^{19}$ performed a retrospective cohort study in patients with first-episode schizophrenia and never previously treated with antipsychotics. They found that after a short period of antipsychotic exposure (22.7 days in average), lipid abnormalities markers were significantly elevated. ${ }^{19}$ Dyslipidaemia induced by antipsychotics was considered as resulting from drug-induced weight gain. ${ }^{26}$ Evidence showed that binding to certain neurotransmitter receptors might partly explain antipsychotic associated weight gain. ${ }^{27}$ For example, olanzapine has relatively high $5-\mathrm{HT}_{2 \mathrm{~A}}$ receptor blocking activity, which increases appetite and may be associated with weight gain. ${ }^{27}$ Besides, drugs with high affinity for $\mathrm{H}_{1}$ receptors have sedative effects, which may induce weight gain because of reduced mobility. ${ }^{27}$ Furthermore, new evidence revealed that dyslipidaemia may occur very early during treatment and may even precede weight gain. ${ }^{26}$ In liver, excess sterol-regulatory element-binding proteins (SREBP) activity could account for elevated circulating cholesterol, free-fatty acids and triacylglycerols. ${ }^{29}$ Animal study suggested that clozapine and risperidone significantly increased hepatic expressions of SREBP in rats, whereas levels of serum lipid parameters were markedly 
elevated. ${ }^{29}$ Therefore, dyslipidaemia should not be viewed only as a consequence of weight gain, but also as a separate and direct adverse effect of antipsychotics therapy. ${ }^{12}$ In this study, inpatients used antipsychotics for more than 8 years had a four times higher risk of dyslipidaemia compared with inpatients never use antipsychotics with similar follow-up time. This particular higher risk might because that the longer the treatment, the greater the chance of insufficient adherence. ${ }^{30}$ Poor adherence to antipsychotic treatments may influence adherence to medications for comorbid health conditions, which may lead to poorly managed and undertreated comorbid conditions in this population. ${ }^{31}$

Our results from subgroup analysis showed that antipsychotics use was associated with higher risk of dyslipidaemia, regardless of the characteristics of inpatients. It is remarkable that the risk of dyslipidaemia associated with antipsychotics was much higher among inpatients aged 18-29 years. This result was in line with that of previous study. ${ }^{26}$ Our result suggested that young mental health inpatients who were between 18 and 29 years old had substantially higher risk for dyslipidaemia after antipsychotics treatment compared with inpatients from other age groups. It was reported that the awareness, treatment and control rates of dyslipidaemia were lower among young population in China. ${ }^{5}$ Therefore, monitoring lipid parameters during antipsychotic treatment should not be restricted to older inpatients, but in all age group inpatients starting antipsychotic medication.

Another important finding in our study was that inpatients without hypertension had substantially higher risk of dyslipidaemia after antipsychotic exposure. The clinical use of antipsychotics should take into consideration the psychiatric and physical condition of the patient and the pharmacological profiles of drugs. ${ }^{12}$ In this study, the baseline prevalence of hypertension was lower in antipsychotic exposed group than that in antipsychotic unexposed group. As hypertension and diabetes were major risk factors of dyslipidaemia, ${ }^{5}$ patients with these conditions might be likely given lower metabolic risk antipsychotics, while patients without baseline hypertension or diabetes might be likely given higher metabolic risk antipsychotics. ${ }^{19}$ Our results suggested that intensive metabolic monitoring and intervention should accompany antipsychotic treatment in all patients, regardless of their baseline comorbid conditions. ${ }^{26}$

Our study has several strengths. First, out study was a large hospital-based cohort study, the long-term follow-up enabled us to detect the long-term effect of antipsychotic treatment. Second, dyslipidaemia cases were identified according to ICD-10 codes from medical records. The results from our study were comparable with other studies of the similar kind. Third, we excluded patients who had dyslipidaemia and fatty liver at baseline, which ensured that newly recorded dyslipidaemia cases during the study period were more likely to be associated with antipsychotics, and reduced the bias of observation study.
Our study has several limitations. First, we did not include outpatients and those treated and managed in communities in the present study. Therefore, the generalisability of our findings might be biased. Second, as patients might use antipsychotics before entering cohort, or discontinue their drug treatment during the study period, the accurate duration of antipsychotics exposure might be different from the follow-up time. Our results using the length of follow-up as proxy for the duration of antipsychotics exposure might be biased. Third, some factors related with dyslipidaemia were not available from medical records, such as body weight, smoking status, diet and physical activity. Thus, we could not control these factors in our analysis, which might bias our results. Finally, antipsychotics were grouped in this study, thus no results about specific antipsychotics could be made. Further studies are required for dyslipidaemia risk associated with specific antipsychotic drugs.

\section{CONCLUSIONS}

Both short-term and long-term antipsychotics use was associated with higher risk of dyslipidaemia among Chinese inpatients with mental illness. Dyslipidaemia risk increased with duration of antipsychotics use. Dyslipidaemia was especially prominent in young patients and those without hypertension. Metabolic monitoring and intervention should accompany antipsychotic treatment in all patients for early detection of lipid abnormality and reduction of cardiovascular risk.

Acknowledgements We would like to thank all patients and health workers in 19 specialised psychiatric hospitals in Beijing. We also thank Beijing Municipal Commission of Health and Family Planning Information Centre for providing the data.

Contributors QM and FY searched the literature, designed the study, analysed the data, interpreted the results, and drafted the manuscript. BM, WJ, JL, MG, JL and ZW collected the data and revised the manuscript. ML conceived the study, designed the study, supervised the study, interpreted the results, and revised the manuscript. All authors contributed to the writing of the manuscript. $\mathrm{ML}$ is the guarantor.

Funding This work was supported by the grant from National Natural Science Foundation of China (grant numbers 71874003, 81703240).

Competing interests None declared.

Patient and public involvement Patients and/or the public were not involved in the design, or conduct, or reporting, or dissemination plans of this research.

Patient consent for publication Not required.

Ethics approval The study used information that is available in database of the Beijing Municipal Commission of Health and Family Planning Information Centre.

Provenance and peer review Not commissioned; externally peer reviewed.

Data availability statement Data may be obtained from a third party and are not publicly available.

Open access This is an open access article distributed in accordance with the Creative Commons Attribution Non Commercial (CC BY-NC 4.0) license, which permits others to distribute, remix, adapt, build upon this work non-commercially, and license their derivative works on different terms, provided the original work is properly cited, appropriate credit is given, any changes made indicated, and the use is non-commercial. See: http://creativecommons.org/licenses/by-nc/4.0/. 
ORCID iD

Min Liu http://orcid.org/0000-0002-5059-3743

\section{REFERENCES}

1 De Hert M, Detraux J, Vancampfort D. The intriguing relationship between coronary heart disease and mental disorders. Dialogues Clin Neurosci 2018;20:31-40

2 Walker ER, McGee RE, Druss BG. Mortality in mental disorders and global disease burden implications: a systematic review and metaanalysis. JAMA Psychiatry 2015;72:334-41.

3 Khan A, Faucett J, Morrison S, et al. Comparative mortality risk in adult patients with schizophrenia, depression, bipolar disorder, anxiety disorders, and attention-deficit/hyperactivity disorder participating in psychopharmacology clinical trials. JAMA Psychiatry 2013;70:1091-9.

4 Correll CU, Detraux J, De Lepeleire J, et al. Effects of antipsychotics, antidepressants and mood stabilizers on risk for physical diseases in people with schizophrenia, depression and bipolar disorder. World Psychiatry 2015;14:119-36.

5 Pan L, Yang Z, Wu Y, et al. The prevalence, awareness, treatment and control of dyslipidemia among adults in China. Atherosclerosis 2016;248:2-9.

6 Opoku S, Gan Y, Fu W, et al. Prevalence and risk factors for dyslipidemia among adults in rural and urban China: findings from the China national stroke screening and prevention project (CNSSPP). BMC Public Health 2019;19:1500.

7 Ventriglio A, Gentile A, Stella E, et al. Metabolic issues in patients affected by schizophrenia: clinical characteristics and medical management. Front Neurosci 2015;9:297.

8 Correll CU, Robinson DG, Schooler NR, et al. Cardiometabolic risk in patients with first-episode schizophrenia spectrum disorders: baseline results from the RAISE-ETP study. JAMA Psychiatry 2014;71:1350-63.

9 Vancampfort D, Firth J, Correll CU, et al. The impact of pharmacological and non-pharmacological interventions to improve physical health outcomes in people with schizophrenia: a metareview of meta-analyses of randomized controlled trials. World Psychiatry 2019;18:53-66.

10 Ruzanna ZZ, Ong LY, Cheah YC, et al. The association between dyslipidaemia and types of antipsychotic medications among patients with chronic schizophrenia. Med J Malaysia 2012;67:39-44.

11 Pillinger T, McCutcheon RA, Vano L, et al. Comparative effects of 18 antipsychotics on metabolic function in patients with schizophrenia, predictors of metabolic dysregulation, and association with psychopathology: a systematic review and network meta-analysis. Lancet Psychiatry 2020;7:64-77.

12 De Hert M, Detraux J, van Winkel R, et al. Metabolic and cardiovascular adverse effects associated with antipsychotic drugs. Nat Rev Endocrinol 2011;8:114-26.

13 Buchanan RW, Kreyenbuhl J, Kelly DL, et al. The 2009 schizophrenia PORT psychopharmacological treatment recommendations and summary statements. Schizophr Bull 2010;36:71-93.

14 Olose EO, Edet J, Igwe MN, et al. Dyslipidaemia and medical outcome (health related quality of life) in patients with schizophrenia taking antipsychotics in Enugu, Nigeria. Psychiatry J 2017;2017:9410575.

15 Birkenaes AB, Birkeland KI, Engh JA, et al. Dyslipidemia independent of body mass in antipsychotic-treated patients under real-life conditions. J Clin Psychopharmacol 2008;28:132-7.

16 Geddes JR, Miklowitz DJ. Treatment of bipolar disorder. Lancet 2013;381:1672-82

17 Zhou X, Keitner Gl, Qin B, et al. Atypical antipsychotic augmentation for treatment-resistant depression: a systematic review and network meta-analysis. Int J Neuropsychopharmacol 2015;18:pyv060.

18 Tiihonen J, Mittendorfer-Rutz E, Majak M, et al. Real-World Effectiveness of Antipsychotic Treatments in a Nationwide Cohort of 29823 Patients With Schizophrenia. JAMA Psychiatry 2017;74:686-93.

19 Zhai D, Cui T, Xu Y, et al. Cardiometabolic risk in first-episode schizophrenia (FES) patients with the earliest stages of both illness and antipsychotic treatment. Schizophr Res 2017;179:41-9.

20 World Health Organization. International statistical classification of diseases and health related problems, 10th revision [Chinese version. Beijing: People's Medical Publishing House, 1996.

21 Chinese guidelines on prevention and treatment of dyslipidemia in adults. Joint Committee for developing Chinese guidelines on prevention and treatment of dyslipidemia in adults. Chinese Journal of Cardiology 2007;35:390-419.

22 Chien I-C, Lin C-H, Chou Y-J, et al. Increased risk of hyperlipidemia in patients with major depressive disorder: a population-based study. $J$ Psychosom Res 2013;75:270-4.

$23 \mathrm{Hsu} \mathrm{J}-\mathrm{H}$, Chien I-C, Lin C-H. Increased risk of hyperlipidemia in patients with bipolar disorder: a population-based study. Gen Hosp Psychiatry 2015;37:294-8.

24 Vancampfort D, Stubbs B, Mitchell AJ, et al. Risk of metabolic syndrome and its components in people with schizophrenia and related psychotic disorders, bipolar disorder and major depressive disorder: a systematic review and meta-analysis. World Psychiatry 2015;14:339-47.

25 Leucht S, Cipriani A, Spineli L, et al. Comparative efficacy and tolerability of 15 antipsychotic drugs in schizophrenia: a multipletreatments meta-analysis. Lancet 2013;382:951-62.

26 Delacrétaz A, Vandenberghe F, Gholam-Rezaee M, et al. Early changes of blood lipid levels during psychotropic drug treatment as predictors of long-term lipid changes and of new onset dyslipidemia. J Clin Lipidol 2018;12:219-29.

27 Nasrallah HA. Atypical antipsychotic-induced metabolic side effects: insights from receptor-binding profiles. Mol Psychiatry 2008;13:27-35.

28 Huang J, Hei G-R, Yang Y, et al. Increased appetite plays a key role in olanzapine-induced weight gain in First-Episode schizophrenia patients. Front Pharmacol 2020;11:739.

29 Cai HL, Tan QY, Jiang P, et al. A potential mechanism underlying atypical antipsychotics-induced lipid disturbances. Trans/ Psychiatry 2015;5:e661.

30 Correll CU, Rubio JM, Kane JM. What is the risk-benefit ratio of longterm antipsychotic treatment in people with schizophrenia? World Psychiatry 2018;17:149-60.

31 Farley JF, Hansen RA, Yu-Isenberg KS, et al. Antipsychotic adherence and its correlation to health outcomes for chronic comorbid conditions. Prim Care Companion CNS Disord 2012;14:PC C. $11 \mathrm{~m} 01324$. 